\title{
Erratum to: Sub-range Jacobi polynomials
}

\section{Walter Gautschi ${ }^{1}$}

Published online: 21 December 2016

(C) Springer Science+Business Media New York 2016

Erratum to: Numer. Algorithms (2012) 61:649-657

DOI 10.1007/s11075-012-9556-z

The first relation in (2.9) should read

$$
\alpha_{k}=\frac{1+c}{2} a_{k}-\frac{1-c}{2}, \quad k \geq 0 .
$$

Walter Gautschi

wgautschi@purdue.edu

1 Department of Computer Sciences, Purdue University, West Lafayette, IN 47907-2066, USA 